\title{
Progressive multifocal leukoencephalopathy: a report of three cases in HIV-negative patients with non-Hodgkin's lymphomas treated with rituximab
}

\author{
Marco Tuccori • Daniele Focosi • Fabrizio Maggi • \\ Mirco Cosottini • Barbara Meini • Fabio Lena • \\ Corrado Blandizzi $\cdot$ Mario Del Tacca $\cdot$ Mario Petrini
}

Received: 7 August 2009 / Accepted: 14 August 2009/Published online: 29 August 2009

(C) Springer-Verlag 2009

\begin{abstract}
Dear Editor,
Progressive multifocal leukoencephalopathy (PML) results from productive infection of glial cells by John Cunningham polyomavirus (JCV), facilitated by immune deficiency [1]. We report three cases of PML in HIV-negative patients who had been treated with rituximab (their main clinical features are summarized in Table 1).
\end{abstract}

Case 1 A 45-year-old male patient with mantle cell lymphoma was treated with four courses of R-hyperCVAD (October 2000-August 2001), followed by mitoxantrone and melphalan supported by autologous hematopoietic stem cell transplantation (HSCT; October 2001). Complete remission was maintained with four courses of rituximab $375 \mathrm{mg} / \mathrm{m}^{2}$ (May-June 2002). On November 2005, a bone relapse required four courses of R-DHAP (ended in February 2006)

Marco Tuccori and Daniele Focosi contributed equally to this manuscript.

\footnotetext{
M. Tuccori $(\bowtie) \cdot$ C. Blandizzi $\cdot$ M. Del Tacca

Division of Pharmacology and Chemotherapy,

Department of Internal Medicine, University of Pisa,

Via Roma 55,

56126 Pisa, Italy

e-mail: m.tuccori@ao-pisa.toscana.it

M. Tuccori $\cdot$ B. Meini $\cdot$ F. Lena $\cdot$ C. Blandizzi $\cdot$ M. Del Tacca

Tuscan Regional Centre for Pharmacovigilance,

Florence, Italy

D. Focosi $\cdot$ M. Petrini

Division of Haematology, Department of Oncology,

Transplantations and New Technologies in Medicine,

University of Pisa,

Pisa, Italy
}

and BEAM-conditioned allogeneic HSCT (March 2006). Graft-versus-host disease prophylaxis was carried out with methotrexate $15 \mathrm{mg} / \mathrm{m}^{2}$ on day +1 and $10 \mathrm{mg} / \mathrm{m}^{2}$ on days $+3,+6$, and +11 , and cyclosporine A titrated to keep serum levels between 200 and $400 \mathrm{pg} / \mathrm{ml}$ until October 2006 . Five courses of maintenance rituximab $\left(375 \mathrm{mg} / \mathrm{m}^{2}\right)$ were then administered (January 2007-April 2008). On April 2008, while in complete remission, he developed bilateral hemianopsia. Magnetic resonance imaging (MRI) displayed subcortical lesions of the white matter. A stereotactic brain biopsy (June 2008) revealed the presence of JCV DNA by polymerase chain reaction (PCR), and histomorphology confirmed PML. Despite treatments with mirtazapine 30-45 mg/day orally, cidofovir $5 \mathrm{mg} / \mathrm{kg}$ intravenously (four courses), and risperidone $4 \mathrm{mg} /$ bid orally, follow-up MRIs (June-September 2008) showed progression of

\section{F. Maggi}

Virology Section and Retrovirus Centre,

Department of Experimental Pathology, University of Pisa, Pisa, Italy

\section{Cosottini}

Department of Neuroscience, University of Pisa, Pisa, Italy

\section{B. Meini $\cdot$ F. Lena}

Pharmaceutical Unit, Hospital of Livorno,

Livorno, Italy 
Table 1 Summary of patients' clinical features

\begin{tabular}{|c|c|c|c|}
\hline Features & Case 1 & Case 2 & Case 3 \\
\hline Age/gender & $45 /$ male & $68 /$ male & 66/male \\
\hline Malignancy & Mantle cell lymphoma & Splenic marginal zone lymphoma & Mantle cell lymphoma \\
\hline $\begin{array}{l}\text { Rituximab exposures } \\
\text { (regimens) }\end{array}$ & $\begin{array}{l}\text { R-hyperCVAD } \\
\text { R-DHAP } \\
\text { Maintenance }\end{array}$ & $\begin{array}{l}\text { R-CVP } \\
\text { Maintenance }\end{array}$ & R-CHOP \\
\hline $\begin{array}{l}\text { Exposure to drugs for } \\
\text { which evidence exists of a } \\
\text { possible association with } \\
\text { PML }\end{array}$ & $\begin{array}{l}\text { Cyclosporine methotrexate } \\
\text { Oral glucocorticoids } \\
\text { (dexamethasone) }\end{array}$ & $\begin{array}{l}\text { Cladribine } \\
\text { Cyclophosphamide } \\
\text { Oral glucocorticoids (prednisone) }\end{array}$ & $\begin{array}{l}\text { Cyclophosphamide } \\
\text { Oral glucocorticoids (prednisone) }\end{array}$ \\
\hline $\begin{array}{l}\text { Other drug/regimen } \\
\text { exposures }\end{array}$ & $\begin{array}{l}\text { Mitoxantrone } \\
\text { Melphalan } \\
\text { BEAM }\end{array}$ & $\begin{array}{l}\text { Bendamustine } \\
\text { Lenalidomide } \\
\text { COMP }\end{array}$ & P-VMyBEC \\
\hline Stem cell transplantation & Allogeneic & Not performed & Not performed \\
\hline $\begin{array}{l}\text { Laboratory analysis at PML } \\
\text { diagnosis }\end{array}$ & $\begin{array}{l}\text { WBC: 7,660; RBC: 5.18; PLT: } \\
\text { 237,000; NEU: 62; LYM: 24; } \\
\text { BC: absent; IgG: 520; IgA: } 22 \text {; } \\
\text { IgM: } 18\end{array}$ & $\begin{array}{l}\text { WBC: 34,000; RBC: 3.49; PLT: } \\
\text { 102,000; NEU: 5; LYM: 18; } \\
\text { BC: 75; IgG: 565; IgA: 109; } \\
\text { IgM: } 21\end{array}$ & $\begin{array}{l}\text { WBC: 23,200; RBC: NA; PLT: } \\
\text { 299,000; NEU: 17; LYM: 74; } \\
\text { BC: absent; IgG: 930; IgA: 198; } \\
\text { IgM: } 50\end{array}$ \\
\hline $\begin{array}{l}\text { PML treatments } \\
\text { (ineffective) }\end{array}$ & $\begin{array}{l}\text { Cidofovir } \\
\text { Mirtazapine } \\
\text { Risperidone }\end{array}$ & Mirtazapine & None \\
\hline
\end{tabular}

$P M L$ Progressive multifocal leukoencephalopathy; $N A$ not available; $W B C$ white blood count (normal values (n.v.): $5,000-10,000 / \mathrm{mm}^{3}$ ); $R B C$ red blood count (n.v.: $4.4-5.6 \times 10^{6} / \mathrm{mm}^{3}$ ); PLT platelets (n.v.: $150,000-400,000 / \mathrm{mm}^{3}$ ); $N E U$ neutrophils (n.v.:40-75\%); $L Y M$ lymphocytes (n.v.: 20-45); $B C$ blast cells (n.v.: 0\%); $\operatorname{Ig} G \mathrm{G}$ immunoglobulins (n.v.: $800-1,800 \mathrm{mg} / \mathrm{dL}$ ); $\operatorname{Ig} A$ A immunoglobulins (n.v.: $90-400 \mathrm{mg} / \mathrm{dL}$ ); $\operatorname{Ig} M \mathrm{M}$ immunoglobulins (n.v.: 60-280 mg/dL); R-hyperCVAD rituximab $375 \mathrm{mg} / \mathrm{m}^{2}$ intravenously (i.v.), cyclophosphamide $300 \mathrm{mg} / \mathrm{m}^{2}$ i.v., doxorubicin $50 \mathrm{mg} / \mathrm{m}^{2}$ i.v., vincristine $2 \mathrm{mg}$ i.v., dexamethasone $40 \mathrm{mg}$ i.v. or orally, G-CSF $10 \mu \mathrm{g} / \mathrm{kg}$, and methotrexate $200 \mathrm{mg} / \mathrm{m}^{2}$ i.v.; $R$-DHAP rituximab $375 \mathrm{mg} / \mathrm{m}^{2}$ i.v. on day 1 , cisplatin $100 \mathrm{mg} / \mathrm{m}^{2}$ i.v. on day 2 , cytosine arabinoside $2 \mathrm{~g} / \mathrm{m}^{2}$ twice daily i.v. on day 3 , and dexamethasone $40 \mathrm{mg}$ i.v. on days 2 through $5 ; R$-CVP rituximab $375 \mathrm{mg} / \mathrm{m}^{2}$ i.v., cyclophosphamide $400 \mathrm{mg} / \mathrm{m}^{2}$ i.v. on days 1 through 5 , vincristine $1.4 \mathrm{mg} / \mathrm{m}^{2}$ i.v. on day 1 , and prednisone $100 \mathrm{mg} / \mathrm{m}^{2}$ orally days 1 through $5 ; B E A M$ carmustine $300 \mathrm{mg} / \mathrm{m}^{2}$ i.v. on day -6 , etoposide $200 \mathrm{mg} / \mathrm{m}^{2}$ i.v., followed by cytarabine $200 \mathrm{mg} / \mathrm{m}^{2}$ i.v. twice daily, on days -5 through -2 , and melphalan $140 \mathrm{mg} / \mathrm{m}^{2}$ i.v. on day $-1 ; C O M P$ cyclophosphamide $750 \mathrm{mg} / \mathrm{m}^{2}$ i.v. on day 1 , doxorubicin $50 \mathrm{mg} / \mathrm{m}^{2}$, vincristine $2 \mathrm{mg}$, and prednisone $100 \mathrm{mg}$ orally on days 1 through 5 ; $R$-CHOP cyclophosphamide $750 \mathrm{mg} / \mathrm{m}^{2}$ i.v. on day 1 , doxorubicin $50 \mathrm{mg} / \mathrm{m}^{2}$ i.v. on day 1 , vincristine $1.4 \mathrm{mg} / \mathrm{m}^{2}$ on day 1 , and prednisone $100 \mathrm{mg}$ orally on day 1 through $5 ; P$-VMyBEC prednisone $50 \mathrm{mg}$ orally, liposomal doxorubicin $30 \mathrm{mg} / \mathrm{m}^{2}$ i.v. bolus, etoposide $100 \mathrm{mg} / \mathrm{m}^{2}$ i.v. on days $1,15,29$, and 43 , cyclophosphamide $350 \mathrm{mg} / \mathrm{m}^{2}$ i.v. on days $1,14,28$, and 42 , vincristine $2 \mathrm{mg}$ i.v. bolus, and bleomycin $5 \mathrm{mg} / \mathrm{m}^{2}$ i.v. bolus on days 7,21 , and 35

demyelinating lesions. The patient died from respiratory failure on September 2008.

Case 2 A 68-year-old male patient received nine courses of intravenous cladribine for splenic marginal zone lymphoma (May-December 2002), achieving complete remission. Because of relapsing disease, he received six courses of R-CVP (November 2006-April 2007). Since a partial response was achieved, he was treated with ten courses of maintenance rituximab (375 mg/m $\mathrm{m}^{2}$; June-October 2007). On November 2007, disease progression prompted a treatment with intravenous bendamustine $90 \mathrm{mg} / \mathrm{m}^{2}$ (five courses). On December 2007, the patient complained of dysmnesia and visual disturbances, and MRI revealed demyelinating lesions suggestive of PML (Fig. 1a-d). Due to lymphoma progression, the patient started lenalidomide $25 \mathrm{mg} /$ day (May-June 2008). Histomorphology and a positive JCV PCR on a stereotactic brain biopsy confirmed PML (June 2008). Treatment with mirtazapine $30 \mathrm{mg} /$ day was then started. On September 2008, a follow-up MRI showed new areas of demyelination, despite a moderate improvement in the original demyelinated area (Fig. 1e-f). The patient then underwent four courses of COMP chemotherapy to treat lymphoma progression. On December 2008, a new MRI evaluation showed further worsening of the disease (Fig. 1g-h). The patient finally died from leukostasis in March 2009.

Case 3 A 66-year-old male patient with leukemic mantle cell lymphoma received two doses of rituximab $\left(375 \mathrm{mg} / \mathrm{m}^{2}\right)$ within CHOP regimen (January-February 2004). Due to occurrence of infusion reactions (fever, chills, and lumbar pain), the last two doses were not administered. As only a partial response was achieved, the patient received eight 

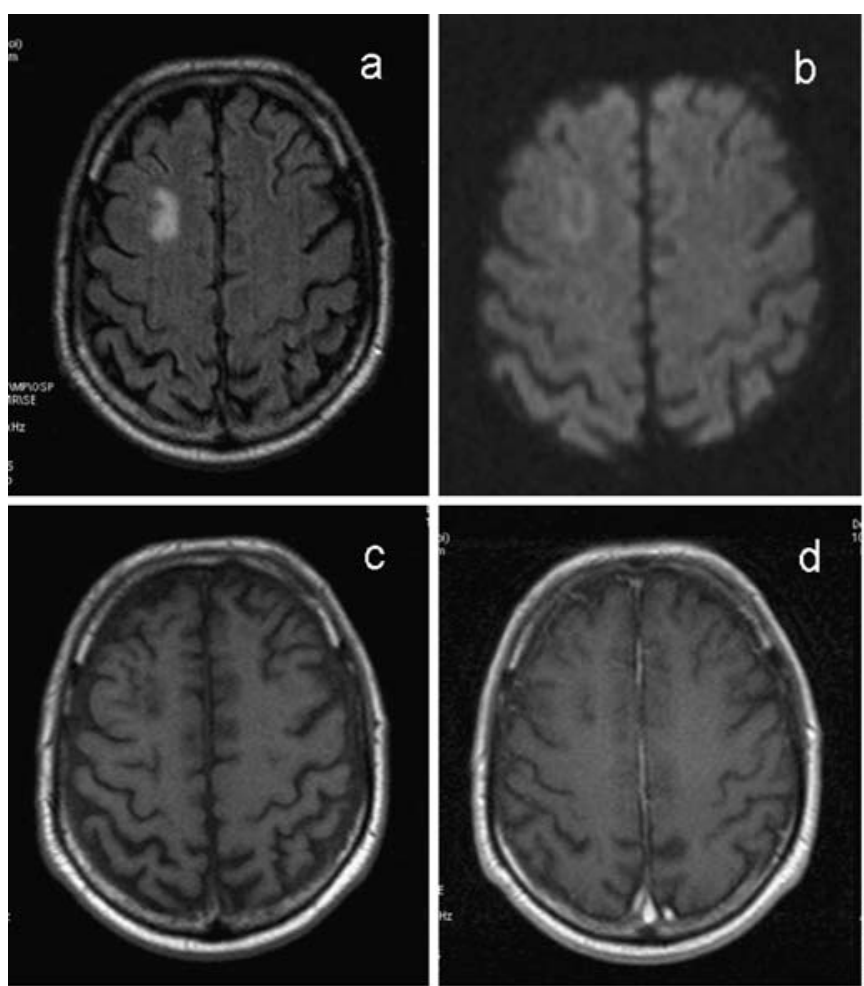

Basal MRI

Fig. 1 MRI in case 2. The first MRI examination (December 2007) revealed a left frontal homogeneous hyperintense lesion of the centrum semiovalis on axial fluid-attenuated inversion recovery (FLAIR) images (a) corresponding to an hypointense (c) nonenhancing (d) area on T1-weighted images. Diffusion-weighted imaging image (b) of the same lesion depicted a central core of hypointensity surrounded by a rim of high signal intensity suggesting a demyelinating lesion
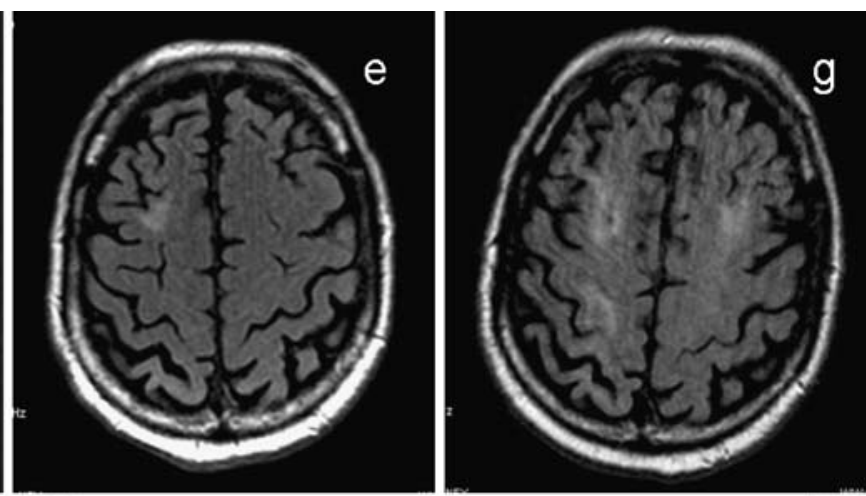

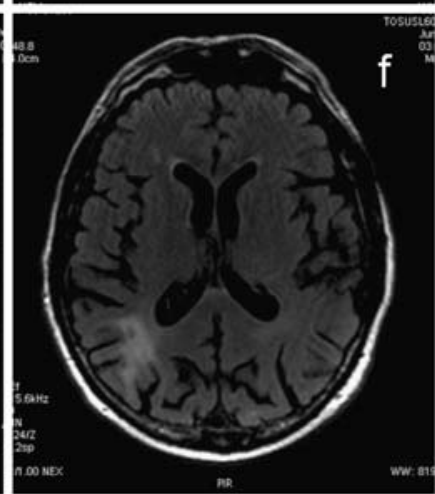

9 Months

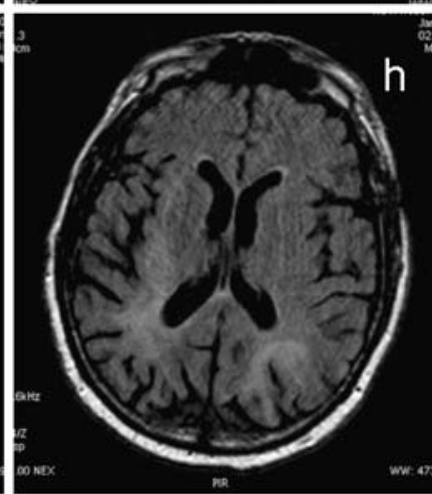

12 Months probably due to PML. Nine months later, the left frontal lesion showed a slight size reduction with atrophic feature of involved cerebral gyri (e). Nevertheless, similar white matter lesions involving also the subcortical "U" fibers with "scalloped" appearance developed in the left temporal and parietal lobes (f). White matter lesions progressively became multiple and confluent, affecting asymmetrically both cerebral hemispheres as shown in axial FLAIR images obtained 12 months later (g-h)

Although immunodeficiency is recognized as a necessary condition for PML development, the mechanism by which JCV crosses the blood-brain barrier (BBB) remains uncertain. Serotonin receptor 2A (5-HT2AR) seems to be involved in JCV internalization into glial cells and some serotonin receptor antagonists (i.e., risperidone, mirtazapine) have been proposed as agents potentially blocking JCV entry $[7,8]$. Two of the present cases received mirtazapine and/or risperidone as a tentative salvage therapy, but these drugs did not provide appreciable benefits. Of note, case 2 showed a delay in the progression of the primary lesion, but new focal lesions developed concomitantly. Consistently with recent investigations $[9,10]$, the unfavorable outcome in our patients suggests that JCV might use alternative gates, besides 5-HT2AR, to cross the BBB.

In conclusion, the present cases support the hypothesis that exposure to rituximab may be an independent risk factor for the development of PML. Moreover, 5-HT2AR antagonists were ineffective in two of our cases, thus pointing out the need for more suitable therapeutic options. 


\section{References}

1. Weber T (2008) Progressive multifocal leukoencephalopathy. Neurol Clin 26:833-854. doi:10.1016/j.ncl.2008.03.007

2. Weber T, Klapper PE, Cleator GM, Bodemer M, Lüke W, Knowles W, Cinque P, Van Loon AM, Grandien M, Hammarin AL, Ciardi M, Bogdanovic G (1997) Polymerase chain reaction for detection of JC virus DNA in cerebrospinal fluid: a quality control study. European Union Concerted Action on Viral Meningitis and Encephalitis. J Virol Methods 69:231-237

3. Cinque P, Koralnik I, Clifford D (2003) The evolving face of human immunodeficiency virus-related progressive multifocal leukoencephalophaty: defining a consensus terminology. J Neurovirol 9(Suppl 1):88-92. doi:10.1080/713831421

4. Pelosini M, Focosi D, Rita F, Galimberti S, Caracciolo F, Benedetti E, Papineschi F, Petrini M (2008) Progressive multifocal leukoencephalopathy: report of three cases in HIV-negative hematological patients and review of literature. Ann Hematol 87:405-412. doi:10.1007/s00277-007-0411-6

5. Carson KR, Evens AM, Richey EA, Habermann TM, Focosi D, Seymour JF, Laubach J, Bawn SD, Gordon LI, Winter JN, Furman RR, Vose JM, Zelenetz AD, Mamtani R, Raisch DW, Dorshimer GW, Rosen ST, Muro K, Gottardi-Littell NR, Talley RL, Sartor O, Green D, Major EO, Bennett CL (2009) Progressive multifocal leukoencephalopathy after rituximab therapy in HIV-negative patients: a report of 57 cases from the Research on Adverse Drug Events and Reports project. Blood 113:4834-4840. doi:10.1182/ blood-2008-10-186999

6. Tuccori M, Focosi D, Blandizzi C, Del Tacca M, Petrini M (2009) Rituximab maintenance for the treatment of patients with follicular lymphoma: systematic review and meta-analysis. J Natl Cancer Inst 110:1288-1289. doi:10.1093/jnci/djp256

7. Elphick GF, Querbes W, Jordan JA, Gee GV, Eash S, Manley K, Dugan A, Stanifer M, Bhatnagar A, Kroeze WK, Roth BL, Atwood WJ (2004) The human polyomavirus, JCV, uses serotonin receptors to infect cells. Science 306:1380-1383. doi:10.1126/ science. 1103492

8. Cettomai D, McArthur JC (2009) Mirtazapine use in human immunodeficiency virus-infected patients with progressive multifocal leukoencephalopathy. Arch Neurol 66:255-258

9. Chapagain ML, Sumibcay L, Gurjav U, Kaufusi PH, Kast RE, Nerurkar VR (2008) Serotonin receptor 2A blocker (risperidone) has no effect on human polyomavirus JC infection of primary human fetal glial cells. J Neurovirol 14:448-454. doi:10.1080/ 13550280802235916

10. Chapagain ML, Verma S, Mercier F, Yanagihara R, Nerurkar VR (2007) Polyomavirus JC infects human brain microvascular endothelial cells independent of serotonin receptor 2A. Virology 364:55-63. doi:10.1016/j.virol.2007.02.018 